Research Article

\title{
Synthesis, Optical, and Magnetic Properties of Graphene Quantum Dots and Iron Oxide Nanocomposites
}

\author{
M. Sajjad $\left(\mathbb{D},{ }^{1,2}\right.$ V. Makarov, ${ }^{2,3}$ M. S. Sultan, ${ }^{3}$ W. M. Jadwisienczak, ${ }^{4}$ B. R. Weiner $\mathbb{D}^{, 2,5}$ \\ and G. Morell id ${ }^{2,3}$ \\ ${ }^{1}$ Western Kentucky University, Bowling Green, KY 42101, USA \\ ${ }^{2}$ Institute of Functional Nanomaterials, University of Puerto Rico, San Juan, PR, USA \\ ${ }^{3}$ Department of Physics, University of Puerto Rico, Rio Piedras Campus, San Juan, PR 00925, USA \\ ${ }^{4}$ School of EECS, Ohio University, Athens, OH 45701, USA \\ ${ }^{5}$ Department of Chemistry, University of Puerto Rico, Rio Piedras Campus, San Juan, PR 00925, USA \\ Correspondence should be addressed to M. Sajjad; muhammad.sajjad@wku.edu
}

Received 16 October 2017; Revised 18 February 2018; Accepted 13 March 2018; Published 12 April 2018

Academic Editor: Raphael Schneider

Copyright (C) 2018 M. Sajjad et al. This is an open access article distributed under the Creative Commons Attribution License, which permits unrestricted use, distribution, and reproduction in any medium, provided the original work is properly cited.

\begin{abstract}
The combination of nanomaterial graphene quantum dots (GQDs) with magnetic nanoparticles offers a unique set of optical and magnetic properties for future energy and medical applications. We report on the synthesis and engineering of GQDs and iron oxide $\left(\mathrm{Fe}_{3} \mathrm{O}_{4}\right)$ nanocomposites (NCs) by using a pulsed laser discharge technique. High-resolution transmission electron microscopy (HRTEM) images showed a high yield of pure GQDs with 2-10 nm diameter. The hexagonal structures and lattice fringes associated with the $\mathrm{C}-\mathrm{C}$ bond in GQDs were clearly identifiable. The structural and optical changes in GQDs and GQDs$\mathrm{Fe}_{3} \mathrm{O}_{4} \mathrm{NC}$ samples induced by UV light were investigated by the absorption and emission spectroscopy over the deep UV-visible spectral range. The photoluminescence spectra have shown subband $\pi \rightarrow \pi^{*}$ transitions in GQDs- $\mathrm{Fe}_{3} \mathrm{O}_{4}$ NC. Magnetic properties of the GQDs- $\mathrm{Fe}_{3} \mathrm{O}_{4} \mathrm{NC}$ samples have shown room temperature ferromagnetism induced by pure $\mathrm{Fe}_{3} \mathrm{O}_{4}$ nanoparticles and from the substantial spin polarized edges of GQD nanoparticles. It is concluded that the observed optical and magnetic properties could be further tailored in the studied nanocomposites for prospective medical applications.
\end{abstract}

\section{Introduction}

Zero-dimensional, graphene quantum dots (GQDs) [1, 2] have shown exceptional physical and chemical properties [3] including, among others, photoluminescence, chemical stability, and pronounced quantum confinement effect making them attractive for novel optoelectronic and energyrelated applications [4]. In addition, due to low toxicity [5], GQDs have significant potential to be used in the field of biophysics and medical sciences as active and passive agents, where they have shown great promise in future therapies for photodynamic treatment of cancerous tumors, imaging, and drug delivery applications [6].

Although nanomaterials including GQDs have already shown numerous properties suitable for biomedical applications $[7,8]$, there still remain substantial complexities involved in handling specific drug delivery dynamics and precise drug dosage control treatments [9]. Some of these complexities can be alleviated by combining the optical properties of GQDs and magnetic properties of iron oxide $\left(\mathrm{Fe}_{3} \mathrm{O}_{4}\right)$ nanocomposites (NCs) [10]. These NCs can be bound to drugs, proteins, enzymes, antibodies, or nucleotides and directed to an organ, tissue, or tumor by using an external magnetic field [10]. Moreover, the magnetic properties of $\mathrm{Fe}_{3} \mathrm{O}_{4}$ nanoparticles make them an excellent contrast agent for MRI applications. However, the limitations of magnetic nanoparticles, including aggregation and precipitation inside the body vessels, can cause serious consequences [11]. As a solution, surface engineering of $\mathrm{Fe}_{3} \mathrm{O}_{4}$ with GQDs was proposed to prevent aggregation and make applications more feasible. On the other hand, GQDs have already shown excellent luminescence response when 


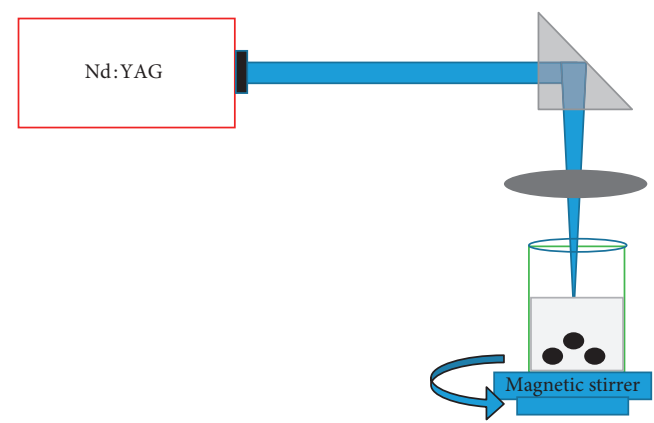

FIGURE 1: Schematic diagram showing synthesis setup for achieving GQDs and GQDs- $\mathrm{Fe}_{3} \mathrm{O}_{4} \mathrm{NC}$.

exposed to the UV light, resulting in visible light emission, whose frequencies depend on the QD's size [12]. Thus, the properties of GQDs can be further tailored by integration with carrier particles (e.g., $\mathrm{Fe}_{3} \mathrm{O}_{4}$ ) as surface decoration or directly embedded into a specific lattice site of NC [13].

In this paper, we report the synthesis and engineering of GQDs- $\mathrm{Fe}_{3} \mathrm{O}_{4} \mathrm{NCs}$ by using a pulsed laser discharge technique in order to tailor the optical and magnetic properties of the material for selected biomedical and/or optoelectronic applications.

\section{Experimental Approach}

The size-selected GQDs and GQDs- $\mathrm{Fe}_{3} \mathrm{O}_{4} \mathrm{NCs}$ were synthesized via a bottom-up approach using pulsed laser-induced irradiation in solution as reported earlier by Habiba et al. [14] with some modifications in experimental parameters. Specifically, the bottom-up method involves photolytic cracking of $\mathrm{C}_{6} \mathrm{H}_{6}$ by laser irradiation in the illuminated region of solution. At the same time, the catalytic particles enhance the interactions between the carbon-containing fragments and stimulate forming $\mathrm{C}-\mathrm{C}$ bonds needed to develop graphene QDs of variable diameter ranging from $2 \mathrm{~nm}$ to $7 \mathrm{~nm}$. Typically, using the selected approach, one can produce GQDs with hydrogen rich surfaces that could be functionalized later with other reagents. In our experiments, first we prepared the mixture of $15 \mathrm{mg}$ nickel (II) oxide (NiO) powder (Alfa Aesar, green), $50 \mathrm{mg}$ polyethlene glycol, and $50 \mathrm{mg}$ iron (II, III) oxide $\left(\mathrm{Fe}_{3} \mathrm{O}_{4}\right)$ powder, respectively. The compounds were ultrasonicated in $20 \mathrm{~mL}$ of benzene (Sigma-Aldrich, anhydrous 99.8\%) for 20 minutes. Next, the solution was irradiated with a laser pulses at 1,064 $\mathrm{nm}$ wavelength generated by Nd:YAG laser (Continuum Surelite, $10 \mathrm{~Hz}, 10 \mathrm{~ns}$ pulse width) as shown in Figure 1. Under these optimized conditions, we obtained a high yield of 1-2 nm GQDs product. The given conditions are also suitable for engineering the GQDs- $\mathrm{Fe}_{3} \mathrm{O}_{4} \mathrm{NC}$.

The typical laser power density was adjusted to $15.9 \times 10^{8} \mathrm{Wcm}^{-2}$, corresponding to an optical energy of $30 \mathrm{~mJ} /$ pulse. Following the synthesis, the residual solid was filtered out from the irradiated mixture leaving behind a solution containing GQD- $\mathrm{Fe}_{3} \mathrm{O}_{4} \mathrm{NC}$ in benzene. This solution was then heated up from room temperature to $370 \mathrm{~K}$ with a heat ramping rate of $10 \mathrm{~K} / \mathrm{min}$ to evaporate solvent and get GQD- $\mathrm{Fe}_{3} \mathrm{O}_{4}$ NC. During the GQD- $\mathrm{Fe}_{3} \mathrm{O}_{4}$ NC separation process, we used a rotary vacuum evaporator to remove the excess benzene. Finally, the GQD- $\mathrm{Fe}_{3} \mathrm{O}_{4} \mathrm{NC}$ was then dissolved in deionized water to produce a concentrated paste for further investigation. The same procedure was repeated to synthesize pure GQDs without $\mathrm{Fe}_{3} \mathrm{O}_{4}$ nanopowder.

Samples were characterized with a high-resolution transmission electron microscope (HRTEM) (JEOL JEM$2200 \mathrm{FS}$ TEM operated at $200 \mathrm{kV}$ ). The UV-visible spectra were recorded on a Varian Cary 1E UV spectrophotometer. For PL characterizations, the excitation and emission spectra of the GQD solutions were recorded using Varian Cary Eclipse with xenon lamp as an excitation source. The magnetic measurements of pure GQDs, iron (II, III) oxide powder, and $\mathrm{Fe}_{3} \mathrm{O}_{4}$-GQD NC were performed using a vibrating-sample magnetometer (VSM, Lake Shore 736) at room temperature. The magnetization $(M)$ versus magnetic field $(H)$ and $M-H$ loops were measured at $300 \mathrm{~K}$ and in the magnetic field range $(15 \mathrm{kOe} \leq H \leq 15 \mathrm{kOe})$, respectively.

\section{Results and Discussion}

The electron microscopy results of as-synthesized pure GQDs and GQDs- $\mathrm{Fe}_{3} \mathrm{O}_{4} \mathrm{NCs}$ are shown in Figures 2(a)2(f). Transmission electron microscope (TEM) images (Figures 2(a)-2(c)) show a uniform dispersion of GQDs, whereas the inset shows the size distribution statistical analysis of as-synthesized GQDs. The experimental conditions were optimized for achieving the GQDs synthesis high yield with a narrow particle size distribution between 1 and $2 \mathrm{~nm}$ diameter. The high resolution TEM (HRTEM) image and the corresponding fast Fourier transform pattern (see the inset in Figure 2(c)) indicate that the GQDs are of a good crystalline quality and have a honeycomb lattice structure, where $\mathrm{C}$ atoms are clearly identifiable (Figure 2(c)). Figures 2(d)-2(f) show the HRTEM images of GQDs- $\mathrm{Fe}_{3} \mathrm{O}_{4}$ $\mathrm{NC}$ collected at $200 \mathrm{~nm}, 50 \mathrm{~nm}$, and $10 \mathrm{~nm}$ scales, respectively. Figure 2(d) shows several dark nanoparticles of $\mathrm{Fe}_{3} \mathrm{O}_{4}$ with diameter less than $100 \mathrm{~nm}$. The magnified image of a selected single $\mathrm{Fe}_{3} \mathrm{O}_{4}$ nanoparticle at scale $50 \mathrm{~nm}$ seen in Figure 2(e) clearly shows several nanoparticles (indicated by arrows) attached to the $\mathrm{Fe}_{3} \mathrm{O}_{4}$ particle surface. The image shown in Figure 2(f) collected at $10 \mathrm{~nm}$ scale identifies that these small particles have carbon traces that confirms that the GQDs are attached to the $\mathrm{Fe}_{3} \mathrm{O}_{4}$ surface.

The structural and optical property changes in NC were studied by monitoring optical absorption and photoluminescence spectra under UV excitation. Figure 3 shows optical absorption spectra due to the $\pi \rightarrow \pi^{*}$ transition in pure GQDs (black curve) peaking at $215 \mathrm{~nm}$ and in GQDs$\mathrm{Fe}_{3} \mathrm{O}_{4} \mathrm{NC}$ (red curve) peaking at $260 \mathrm{~nm}$, respectively. The spectral shift observed in the absorption band peak positions is probably due to electronic transitions between GQDs and $\mathrm{Fe}_{3} \mathrm{O}_{4}$ particles. Furthermore, the shape of the GQDs- $\mathrm{Fe}_{3} \mathrm{O}_{4}$ $\mathrm{NC}$ absorption band is complex due to overlap with sharp peaks observed at $245 \mathrm{~nm}, 250 \mathrm{~nm}, 255 \mathrm{~nm}$, and $260 \mathrm{~nm}$. We assign these sharp peaks to the $\pi \rightarrow \pi^{*}$ transition vibration subbands between $\left(\mathrm{C}_{6} \mathrm{H}_{6}\right)_{n}$ and $\mathrm{Fe}_{3} \mathrm{O}_{4}$ nanoparticle. The inset in Figure 3(a) shows optical images of GQDs and GQDs- $\mathrm{Fe}_{3} \mathrm{O}_{4}$ aqueous solution (DI water) illuminated under 


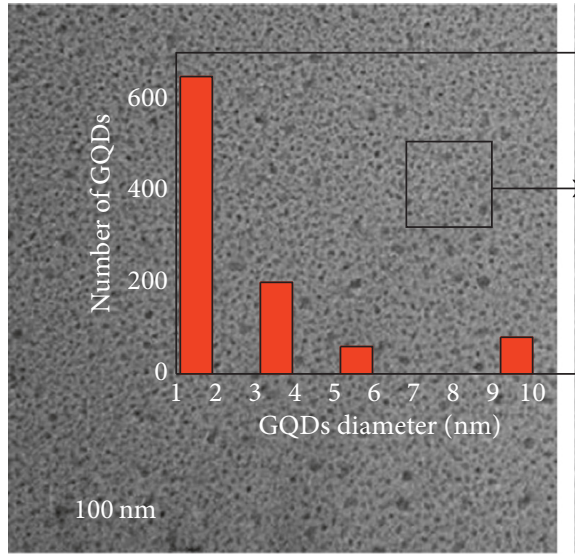

(a)

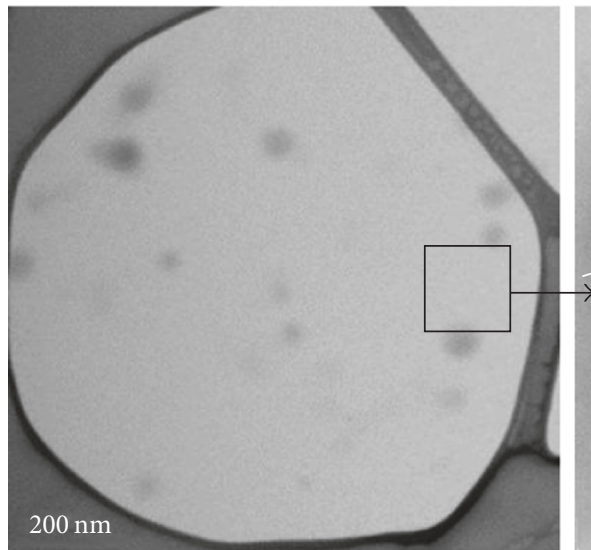

(d)

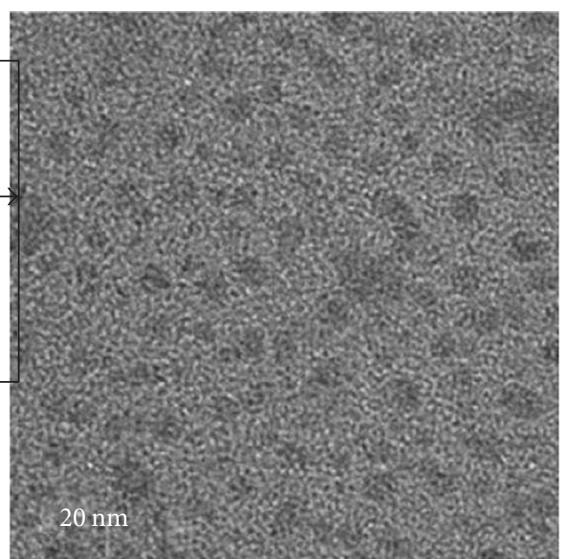

(b)

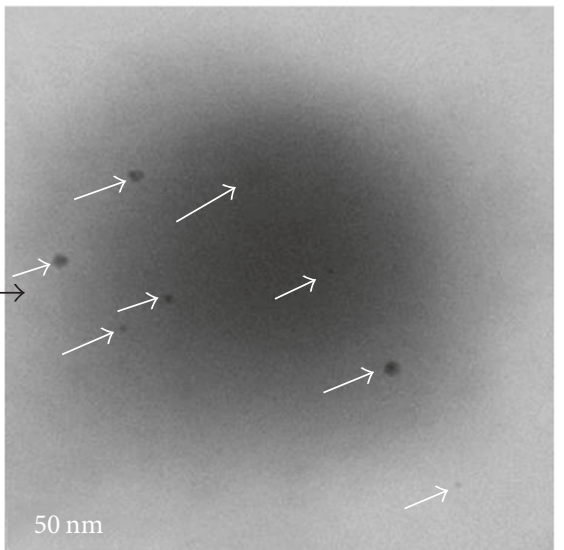

(e)

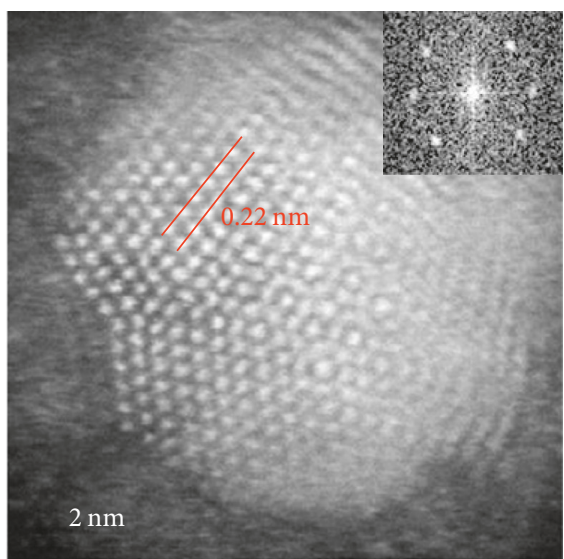

(c)

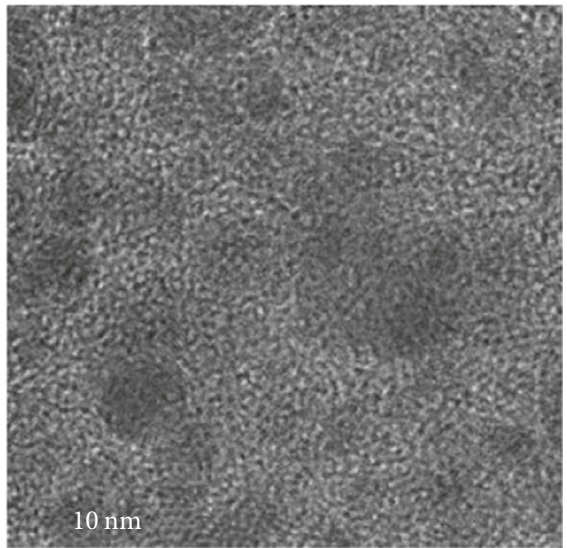

(f)

FIgURE 2: ( $\mathrm{a}, \mathrm{b}, \mathrm{c})$ HRTEM image of pure GQDs and GQDs size hexagonal structure. (d, e, f) HRTEM images of GQDs-Fe $\mathrm{O}_{4} \mathrm{NC}$ discussed in the text. Arrows in (e) indicate a GQD decorated with $\mathrm{Fe}_{3} \mathrm{O}_{4}$ particles seen as dark dots.

UV light. It is seen that, under $365 \mathrm{~nm}$ excitation, the emitted light color from the GQD dispersion changes to blue-green suggesting the existence of transition states between iron core particle and the GQDs attached to $\mathrm{Fe}_{3} \mathrm{O}_{4}$ [15]. Figure 3 (b) shows the photoluminescence (PL) spectra of studied samples when excited at $450 \mathrm{~nm}$. The resulting PL spectra of GQDs dispersion are composed of three subbands peaking at $536 \mathrm{~nm}, 558 \mathrm{~nm}$, and $580 \mathrm{~nm}$, respectively, corresponding to radiative emission from GQDs having different sizes [16]. In the case of GQDs- $\mathrm{Fe}_{3} \mathrm{O}_{4} \mathrm{NC}$, the luminescence intensity doubles, and the PL spectrum shape is broadened and evolved as compared to the GQDs counterpart [17-19]. Moreover, the observed blue shift effect can be assigned to the GQD $\pi$-system polarization effects induced by $\mathrm{Fe}_{3} \mathrm{O}_{4}$ nanoparticle combined with the GQD system. Since $\mathrm{Fe}_{3} \mathrm{O}_{4}$ has a higher electron affinity than GQD, the induced polarization effect creates an increased energy gap in optically active GQD; that is, such an effect induces a blue shift.

The three emission subbands observed for GDQs appeared in GQDs- $\mathrm{Fe}_{3} \mathrm{O}_{4} \mathrm{NC}$ also but at slightly different wavelengths (Figure 3(b)) which confirm that they originate from pure GQDs. An extra subband component appeared at $568 \mathrm{~nm}$ which was not previously observed and is assigned to the $\pi \rightarrow \pi^{*}$ transition in GQDs- $\mathrm{Fe}_{3} \mathrm{O}_{4} \mathrm{NC}$. Furthermore, the observed PL spectra can be considered to arise from transitions from the lowest unoccupied molecular orbital in $\mathrm{Fe}_{3} \mathrm{O}_{4}$ to the highest occupied molecular orbital in GQDs. We conclude that, in both cases, the observed PL spectra are assigned to $\pi \rightarrow \pi^{*}$ transitions; however in the case of NC, this transition can be also affected by an increase in the absorption cross section due to the interaction between GQDs and $\mathrm{Fe}_{3} \mathrm{O}_{4}$ in NC. This assumption is based on the fact that since the physical and chemical properties of GQDs$\mathrm{Fe}_{3} \mathrm{O}_{4} \mathrm{NC}$ change considerably at the nanoscale level, the optical properties also change. It was shown that not only the size of the polymeric structures within the quantum dots can change their emissions, but also surface functionalization can tune resulting luminescence through modulating material band gap [20,21]. Therefore, one can conclude that GQDs possess a tunable energy band gap where the shift in the spectral position can be controlled by attaching GQDs to different nanoparticles [22].

We conclude that such materials might be useful in medical treatments [23] relying on the facts that graphenebased nanomaterials have already shown strong optical absorption in the near-infrared region, and that the GQDs$\mathrm{Fe}_{3} \mathrm{O}_{4} \mathrm{NC}$ are optically active. Therefore, heat can be generated efficiently under light irradiation. Thus, raising temperature at 


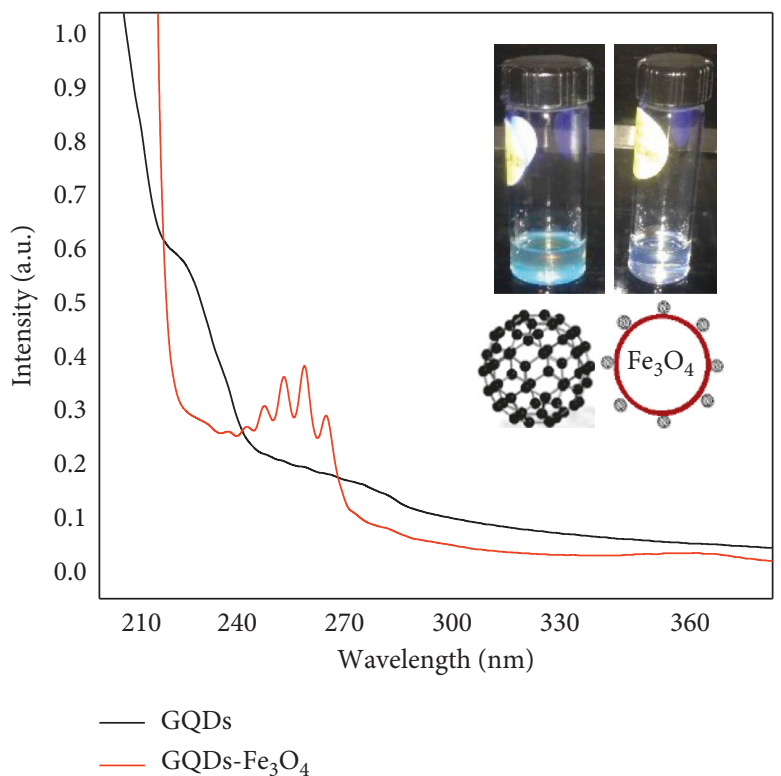

(a)
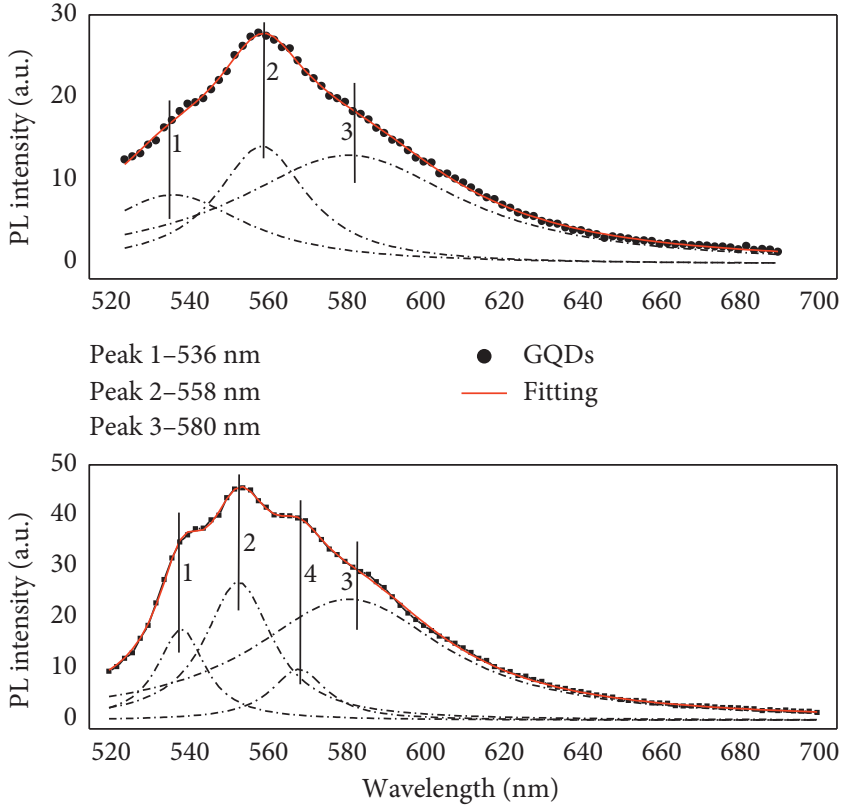

Peak $1-538 \mathrm{~nm}$ Peak $2-552 \mathrm{~nm}$ Peak 3-581 nm
Peak 4-568 nm

- GQDs- $\mathrm{Fe}_{3} \mathrm{O}_{4}$

— Fitting

(b)

FIgURe 3: (a) Room temperature UV-visible absorption spectra of GQDs (black) and GQDs- $\mathrm{Fe}_{3} \mathrm{O}_{4}$ (red). Inset in (a) shows optical images of the emitting GQDs and GQDs- $\mathrm{Fe}_{3} \mathrm{O}_{4}$ aqueous solution (DI water) when excited at $365 \mathrm{~nm}$. (b) Room temperature PL spectra of GQDs (upper panel) and GQDs- $\mathrm{Fe}_{3} \mathrm{O}_{4} \mathrm{NC}$ (lower panel) excited at $450 \mathrm{~nm}$.

tissue sites by using absorbing NC can promote the selective destruction of affected cells when used for the photothermal therapy [23].

The optical spectrum of $\mathrm{Fe}_{3} \mathrm{O}_{4}$ was not recorded because this compound is not soluble in solvents used in our study-it forms a suspension, where $\mathrm{Fe}_{3} \mathrm{O}_{4}$ particles can be considered as black bodies with respect to the optical absorption properties of an active medium. Therefore, the light absorption efficiency is the same across the scanned spectral range, and it is free from any characteristic absorption bands. A small deviation from the black body approximation may give some scattering effects; however, this was not considered here. Anyhow, $\mathrm{Fe}_{3} \mathrm{O}_{4}$ material does not show any emission by itself; however, the GQDs- $\mathrm{Fe}_{3} \mathrm{O}_{4} \mathrm{NC}$ generates interesting optical features as presented above.

We have studied the magnetic properties of GQDs, GQDs- $\mathrm{Fe}_{3} \mathrm{O}_{4}$, and $\mathrm{Fe}_{3} \mathrm{O}_{4}$ magnetic nanoparticles involved in the synthesized NCs. We observed that the $\mathrm{Fe}_{3} \mathrm{O}_{4}$ powder with particle size between 50 and $60 \mathrm{~nm}$ exhibits a strong magnetization curve as shown in Figure 4(a). It is seen that the magnetization curve of a $\mathrm{Fe}_{3} \mathrm{O}_{4}$ sample shows a hysteresis loop; that is, nanoparticles of interest maintain the domain structure and thus is considered as ferromagnetic. Analysis of the respective magnetization hysteresis loop indicated that its magnetic permeability is 6,487 , which is in acceptable agreement with the reported earlier value of 6,783 [24].

The pure GQDs magnetization curve is shown in Figure 4(b), and it does not demonstrate any hysteretic behavior. The magnetization curve measured at $300 \mathrm{~K}$ is practically saturated. Since samples were prepared under an atmosphere containing oxygen, we may not exclude the presence of some parasitic oxide phase which may contribute to the overall magnetic response of the studied samples. On the other hand, the zigzag edge atoms, adatoms, or vacancies are all believed to cause magnetic moments in graphene [25]. Because of these facts, the observed magnetic phenomenon cannot be explained unambiguously by applying a simple paramagnetic model, that is, the Brillouin relationship for magnetization:

$$
\mu=N g_{\mathrm{S}} \mu_{\mathrm{B}}\left[\frac{2 s+1}{2 s} \operatorname{coth}\left(\frac{2 s+1}{2 s} \cdot \frac{g_{\mathrm{S}}\left|\mu_{\mathrm{B}}\right| H_{\mathrm{z}}}{k_{\mathrm{B}} T}\right)-\frac{1}{2 s} \operatorname{coth}\left(\frac{1}{2 s} \cdot \frac{g_{\mathrm{S}}\left|\mu_{\mathrm{B}}\right| H_{\mathrm{z}}}{k_{\mathrm{B}} T}\right)\right] \text {, }
$$

where $N$ is the number of paramagnetic centers per gram of the sample, $g_{\mathrm{s}}$ is the electron spin factor (the Landé factor), $\mu_{\mathrm{B}}$ is the Bohr magneton, $k_{\mathrm{B}}$ is the Boltzmann constant, $T$ is the absolute temperature, $H_{\mathrm{z}}$ is the magnetic field directed along the $z$ axis of the reference-fixed frame, and $s=1 / 2$. To explain the observed phenomenon, we proposed that some 


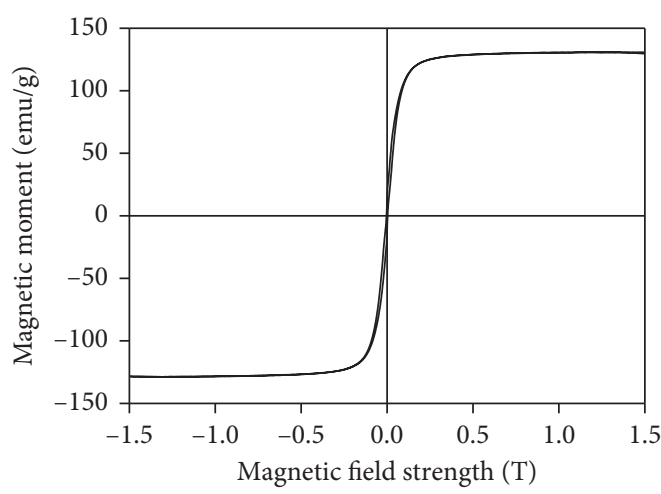

(a)

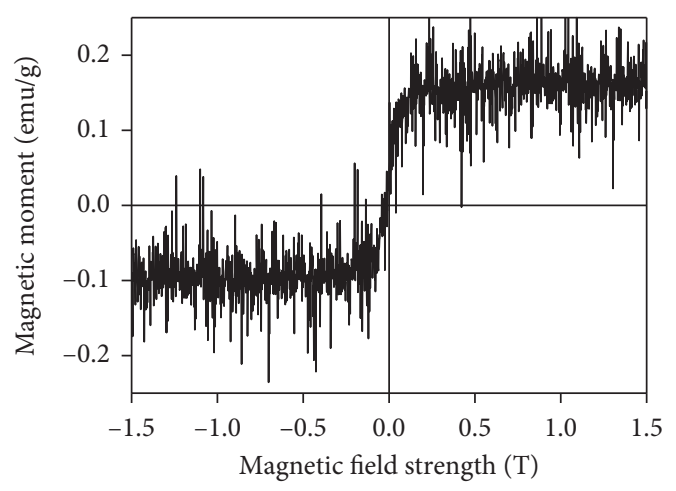

(b)

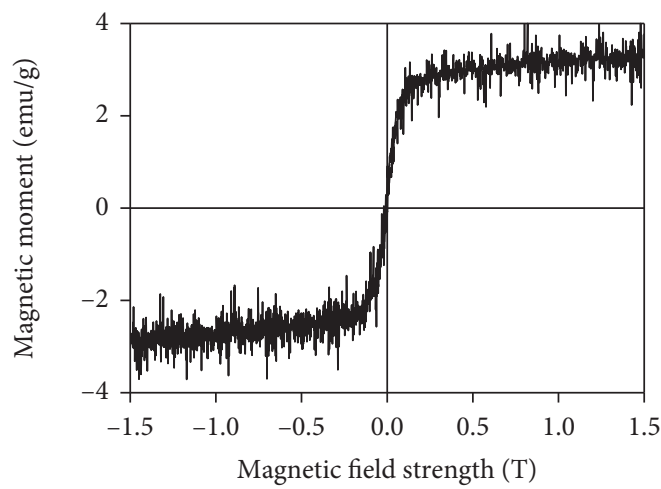

(c)

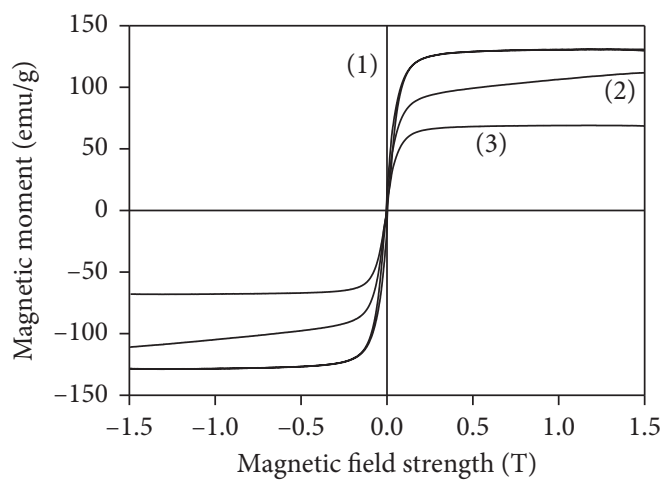

(d)

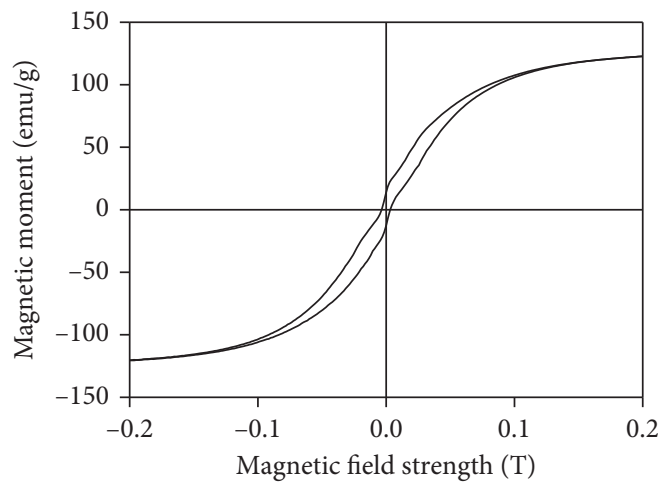

(a')

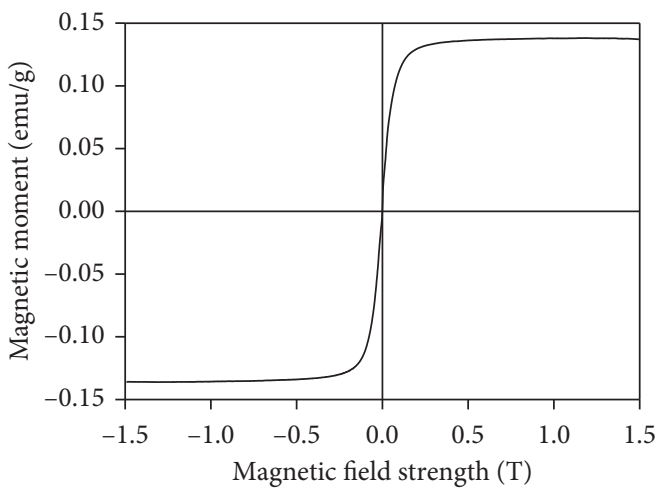

$\left(b^{\prime}\right)$

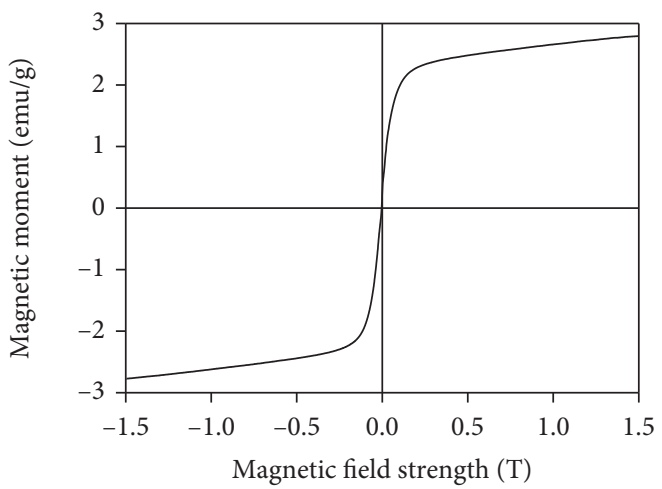

( $\left.c^{\prime}\right)$

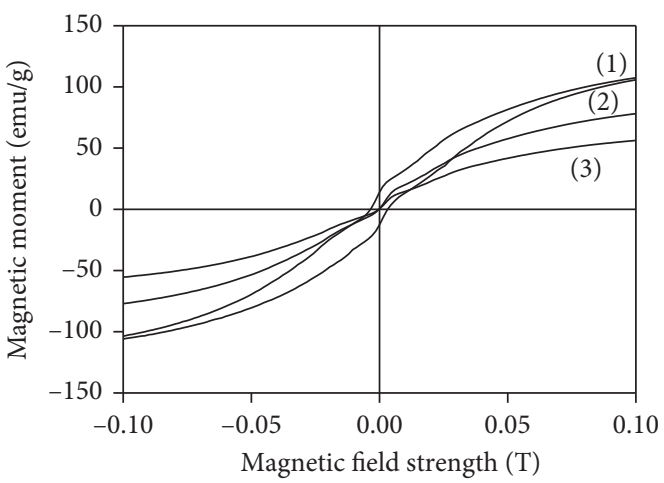

$\left(d^{\prime}\right)$

Figure 4: Experimental magnetization curves of $\mathrm{Fe}_{3} \mathrm{O}_{4}$ powder (a), GQDs (b), and GQD- $\mathrm{Fe}_{3} \mathrm{O}_{4} \mathrm{NC}(\mathrm{c})$ while $\left(\mathrm{a}^{\prime}\right)$, $\left(\mathrm{b}^{\prime}\right)$, and $\left(\mathrm{c}^{\prime}\right)$ panels correspond to fitting curves using (2). ( $\left.d, \mathrm{~d}^{\prime}\right)$ direct comparison and fitting data showing magnetic properties of reference $\mathrm{Fe}_{3} \mathrm{O}_{4}$ powder data (curve 1), GQD- $\mathrm{Fe}_{3} \mathrm{O}_{4}$ composite (curve 2, data factorized with factor 40), and pure GQD (curve 3, data factorized with factor 800). 
of the paramagnetic centers in the GQD particle are coupled by strong exchange interactions, creating a ground electronic state of such system with total spin angular momentum of $S=n s$, where $n$ is the number of centers in the GQD particle and $s=1 / 2$. To analyze the magnetization curve for the materials under consideration, we adopted an approach based on the Zeeman sublevels for spin angular momentum $S$, where the lowest energy level has a spin angular momentum projection $M_{S}=-S$ on the field direction. We were then able to determine the magnetic moment of sample for $S \gg 1$ using the following equation:

$$
\begin{aligned}
& \mu= n_{0} g_{\mathrm{S}} \mu_{\mathrm{B}}\left(1-e^{-\left(\left(g_{\mathrm{S}}\left|\mu_{\mathrm{B}}\right| H_{\mathrm{z}}\right) / k_{\mathrm{B}} T\right)}\right) \\
& \cdot \sum_{M_{\mathrm{S}}=-S}^{M_{\mathrm{S}}=+S} M_{\mathrm{S}} e^{-\left(\left(g_{\mathrm{S}}\left|\mu_{\mathrm{B}}\right|\left(S+M_{\mathrm{S}}\right) H_{\mathrm{z}}\right) / k_{\mathrm{B}} T\right)},
\end{aligned}
$$

where $g_{\mathrm{s}}$ is the electron $g$-factor, $\mu_{\mathrm{B}}$ is the Bohr magneton, $H_{\mathrm{z}}$ is the auxiliary magnetic field, $S$ is the total spin number, and other parameters are as defined above. Taking into account (2), we fitted the magnetization data for GQDs shown in Figure 4(b) and obtained an acceptable fit, shown in Figure $4\left(b^{\prime}\right)$, for $S=217 \pm 6$. On the other hand, we have to assume that "averaged" GQDs consist of 434 paramagnetic centers strongly coupled by an exchange interaction [26]. Taking into account the averaged GQD size of $2-4 \mathrm{~nm}$ and carbon (C) atom radius $0.075 \mathrm{~nm}$, we estimate the number of $\mathrm{C}$ atoms in a typical GQD to be about 2,844 . Estimated magnetic permeability for such a sample is then close to $4.8 \pm 1.3$. Data shown in Figure 4(c) for combined GQD- $\mathrm{Fe}_{3} \mathrm{O}_{4} \mathrm{NC}$ with averaged size of $15 \mathrm{~nm}$ do not demonstrate magnetization hysteresis behavior. In order to explain the data shown in Figure 4(c), we used the same theoretical approach developed above for pure GQD. We applied (2) to fit data shown in Figure 4(c) and obtained an acceptable fit as represented in Figure $4\left(c^{\prime}\right)$. This fitting curve gives $S=673 \pm 12$ where 1,346 unpaired spins in GQD$\mathrm{Fe}_{3} \mathrm{O}_{4} \mathrm{NC}$ are strongly coupled by the exchange interaction and the expected ground state of such NC shall have a multiplicity of 1,347 . The estimated magnetic permeability of GQD- $\mathrm{Fe}_{3} \mathrm{O}_{4} \mathrm{NC}$ is about $124 \pm 8$. Figures $4(\mathrm{~d})$ and $4\left(\mathrm{~d}^{\prime}\right)$ shows a direct comparison between the experimental data and the theoretical fits with respect to the referenced magnetic properties of $\mathrm{Fe}_{3} \mathrm{O}_{4}$ powder (curve a), GQD- $\mathrm{Fe}_{3} \mathrm{O}_{4}$ NC (curve b, data factorized with factor 40), and pure GQD (curve c, data factorized with factor 800), respectively.

It was shown by Sun et al. [27] that pure GQDs demonstrate diamagnetic and paramagnetic properties at low temperature. In their case, the magnetization curve measured at $2 \mathrm{~K}$ demonstrated a saturation effect, and it was fit with high accuracy by (1) where the value of the fitting parameter $J$ was about 0.5 , that is, with high accuracy equal to the electron spin angular momentum. Those data differ from the data reported here because these effects can be neglected at $300 \mathrm{~K}$. We believe that the observed difference can be explained based on the GQD structure at hand. In the present case, the materials synthesis process is carried out in air at atmospheric pressure resulting, possibly, in GQD-O structure formation what ultimately can result in high

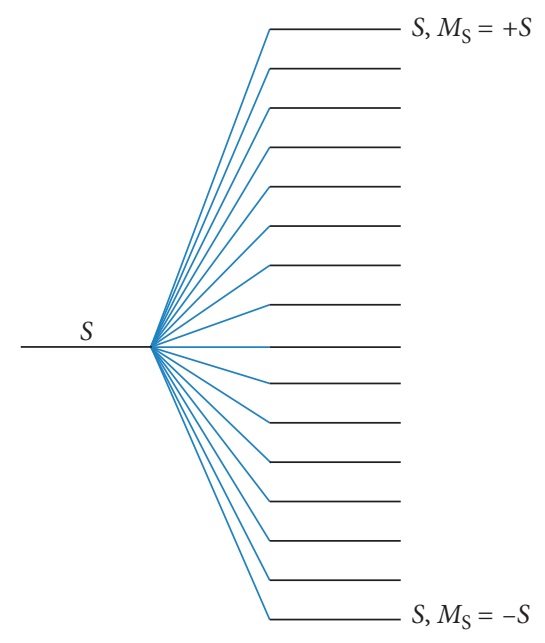

FIGURE 5: Schematic representation of the Zeeman state structure for system with total spin angular momentum $S$ in the presence of an external magnetic field $H$.

exchange interaction between the paramagnetic centers of this material. Figure 4 shows the corresponding magnetization fits for $\mathrm{Fe}_{3} \mathrm{O}_{4}$ nanopowder, GQD-Fe $\mathrm{O}_{4}$ composite, and pure GQDs, respectively. We factorized the data with factor of 40 for GQDs- $\mathrm{Fe}_{3} \mathrm{O}_{4}$ and with the factor of 800 for GQD in order to enhance their relative intensity as compared to reference pure $\mathrm{Fe}_{3} \mathrm{O}_{4}$ nanopowder. It is clearly seen that the GQDs- $\mathrm{Fe}_{3} \mathrm{O}_{4} \mathrm{NCs}$ exhibit strong enhancement of their magnetic moment as compared to pure GQDs. Therefore, we have concluded here that the magnetic properties of studied NCs can be useful for guiding delivery and targeting the specific tissues and cells using an external magnetic field.

By engineering $\mathrm{Fe}_{3} \mathrm{O}_{4}$ with GQDs NCs where optical and magnetic properties are linked together, a new generation of theranostic functionalities can be developed. Such novel hybrid NCs will be suitable for delivering nanocarriers to the tumor sites for imaging, whereas the NCs optical feature can be used as photothermal therapy agents to kill cancer cells efficiently [28-30]. Therefore, the NCs offering both optical and magnetic capabilities may provide an interesting approach for the future medical treatments.

We have grown highly crystalline, honeycomb lattice structure of 2-10 nm GQDs using a pulsed laser photolysis technique in a bottom-up approach. It was shown that the GQDs are luminescent in the green spectral region and that their optical properties can be further tailored by engineered GQDs- $\mathrm{Fe}_{3} \mathrm{O}_{4} \mathrm{NC}$. The pure and engineered quantum dot samples showed different emission spectra due to the subband $\pi \rightarrow \pi^{*}$ transitional states between GQDs and $\mathrm{Fe}_{3} \mathrm{O}_{4}$ nanoparticles. GQDs- $\mathrm{Fe}_{3} \mathrm{O}_{4} \mathrm{NCs}$ have shown ferromagnetism originating partially from pure $\mathrm{Fe}_{3} \mathrm{O}_{4}$ nanopowder and from the substantial spin polarized edges of GQDs. We have demonstrated that not only the QD's size but also the surface functionalization of nanomaterials with GQDs can tune the optical properties of the nanomaterials. Such properties of the functionalized nanocompounds can lead, among others, to novel electronic, energy-related, and advanced biomedical 
applications including cancer treatments, tumor imaging, and dose delivery to the desired cell or tissue for a successful therapeutic effect.

\section{Appendix}

Diagram of the Zeeman sublevels for system with spin angular momentum $S$ is shown in Figure 5 where lowest energy level has spin angular momentum projection on the field direction $-S$. Energy of Zeeman sublevel is determined as follows:

$$
E\left(S, M_{\mathrm{S}}\right)=g_{\mathrm{S}}\left|\mu_{\mathrm{B}}\right| M_{\mathrm{S}} H_{\mathrm{Z}}
$$

where meaning of all parameters included in this relationship is defined in the text. If we will assign to lowest by energy Zeeman level $\left(M_{\mathrm{S}}=-S\right)$ zero energy, energy of spin states will be redetermined as follows:

$$
E\left(S, M_{\mathrm{S}}\right)=g\left|\eta_{\mathrm{B}}\right|\left(S+M_{\mathrm{S}}\right) H_{\mathrm{z}} \text {. }
$$

Taking into account the Boltzmann distribution, population of Zeeman spin states can be defined as follows:

$$
n\left(S, M_{\mathrm{S}}\right)=n_{0} e^{-\left(\left(E\left(S, M_{\mathrm{S}}\right)\right) / k_{\mathrm{B}} T\right)}=n_{0} e^{-\left(\left(g\left|\eta_{\mathrm{B}}\right|\left(S+M_{\mathrm{S}}\right) H_{\mathrm{z}}\right) / k_{\mathrm{B}} T\right)},
$$

where $n_{0}$ was defined in the text as number density of GQD$\mathrm{Fe}_{3} \mathrm{O}_{4}$ composite species. Therefore, magnetic moment " $\mu$ " of one nanocrystal is determined as follows:

$$
\mu=g_{\mathrm{S}} \mu_{\mathrm{B}} \frac{\sum_{M_{\mathrm{S}}=-S}^{M_{\mathrm{S}}=S} M_{\mathrm{S}} n\left(S, M_{\mathrm{S}}\right)}{\sum_{M_{\mathrm{S}}=-S}^{M_{\mathrm{S}}=S} n\left(S, M_{\mathrm{S}}\right)} .
$$

Or, for $S \gg 1$, it can be represented as follows:

$$
\mu=g_{\mathrm{S}} \mu_{\mathrm{B}}\left(1-e^{-\left(\left(g\left|\eta_{\mathrm{B}}\right| H_{\mathrm{z}}\right) / k_{\mathrm{B}} T\right)}\right) \sum_{M_{\mathrm{S}}=-S}^{M_{\mathrm{S}}=S} M_{\mathrm{S}} n\left(S, M_{\mathrm{S}}\right)
$$

Thus, reduced material magnetization (emu/g) can be defined as follows:

$$
M=n_{0} \mu .
$$

For case of $\left(\left(g\left|\mu_{\mathrm{B}}\right| H_{\mathrm{z}}\right) / k_{\mathrm{B}} T\right) \ll 1$, factor in front of sum (A.5) can be rewritten as follows:

$$
\left(1-e^{-\left(\left(g\left|\eta_{\mathrm{B}}\right| H_{\mathrm{z}}\right) / k_{\mathrm{B}} T\right)}\right) \approx \frac{g\left|\mu_{\mathrm{B}}\right| H_{\mathrm{z}}}{k_{\mathrm{B}} T} .
$$

Therefore, (A.5) can be represented as follows:

$$
\mu=\frac{\left(g\left|\mu_{\mathrm{B}}\right|\right)^{2} H_{\mathrm{z}}}{k_{\mathrm{B}} T} \sum_{M_{\mathrm{S}}=-S}^{M_{\mathrm{S}}=S} M_{\mathrm{S}} n\left(S, M_{\mathrm{S}}\right)
$$

\section{Conflicts of Interest}

The authors declare that they have no conflicts of interest.

\section{Acknowledgments}

This project was partially supported by the Institute for Functional Nanomaterials (NSF Grant 1002410), PR NASA
Space Grant (NASA Grant no. NNX15AI11H), and PR NASA EPSCoR (NASA Grant no. NNX15AK43A). The authors would like to acknowledge the Center for Electrochemical Engineering Research (CEER) through the award NSF-MRI no. CBET-1126350 for the access to TEM facility at Ohio University. W. M. Jadwisienczak acknowledges the support from the NSF CAREER program under no. DMR1056493. M. Sajjad would like to recognize Mr. Oscar Resto for TEM analysis support in the Nanoscopy Facility at the University of Puerto Rico, San Juan, Puerto Rico. Also, M. Sajjad thanks Western Kentucky University, Bowling Green, KY, for providing experimental and characterization facilities for data analysis. The TEM analysis was conducted by Yuxuan Wang currently at The University of Texas at Dallas.

\section{References}

[1] J. Zhang, Y.-Q Ma, N. Li et al., "Preparation of graphene quantum dots and their application in cell imaging," Journal of Nanomaterials, vol. 2016, Article ID 9245865, 9 pages, 2016.

[2] C. K. Chua, Z. Sofer, P. Simek et al., "Synthesis of strongly fluorescent graphene quantum dots by cage-opening buckminsterfullerene," ACS Nano, vol. 9, no. 3, pp. 2548-2555, 2015.

[3] N. Suzuki, Y. Wang, P. Elvati et al., "Chiral graphene quantum dots," ACS Nano, vol. 10, no. 2, pp. 1744-1755, 2016.

[4] I. Ozfidan, A. D. Güçlü, M. Korkusinski, and P. Hawrylak, "Theory of optical properties of graphene quantum dots," Physica Status Solidi (RRL)-Rapid Research Letters, vol. 10, no. 1, pp. 102-110, 2016.

[5] S. Wang, I. S. Cole, and Q. Li, "The toxicity of graphene quantum dots," RSC Advances, vol. 6, no. 92, pp. 89867-89878, 2016.

[6] X. Yan, X. Cui, B. Li, and L.-S. Li, "Large, solution-processable graphene quantum dots as light absorbers for photovoltaics," Nano Letters, vol. 10, no. 5, pp. 1869-1873, 2010.

[7] M. Colombo, S. C. Romero, M. F. Casula et al., "Biological applications of magnetic nanoparticles," Chemical Society Reviews, vol. 41, no. 11, pp. 4306-4334, 2012.

[8] Z. Wang, H. Zeng, L. Sun, and J. Mater, "Graphene quantum dots: versatile photoluminescence for energy, biomedical, and environmental applications," Journal of Materials Chemistry C, vol. 3, no. 6, pp. 1157-1165, 2015.

[9] X. Wang, X. Sun, J. Lao et al., "Multifunctional graphene quantum dots for simultaneous targeted cellular imaging and drug delivery," Colloids and Surfaces B: Biointerfaces, vol. 122, pp. 638-644, 2014.

[10] A. K. Gupta and M. Gupta, "Synthesis and surface engineering of iron oxide nanoparticles for biomedical applications," Biomaterials, vol. 26, no. 18, pp. 3995-4021, 2005.

[11] N. Alegreta, A. Criadoa, and M. Pratoto, "Recent advances of graphene-based hybrids with magnetic nanoparticles for biomedical applications," Current Medicinal Chemistry, vol. 24, no. 5, pp. 529-536, 2017.

[12] L. Tang, R. Ji, X. Li, K. S. Teng, and S. P. Lau, "Size-dependent structural and optical characteristics of glucose-derived graphene quantum dots," Particle \& Particle Systems Characterization, vol. 30, no. 6, pp. 523-531, 2013.

[13] D. Qu, M. Zheng, J. Li, Z. Xie, and Z. Sun, "Tailoring color emissions from N-doped graphene quantum dots for bioimaging applications," Light: Science and Applications, vol. 4, no. 12, pp. 1-8, 2015. 
[14] K. Habiba, V. I. Makarov, J. Avalos, M. J.F. Guinel, B. R. Weiner, and G. Morell, "Luminescent graphene quantum dots fabricated by pulsed laser synthesis," Carbon, vol. 64, pp. 341-350, 2013.

[15] J. Shen, Y. Zhu, X. Yang, and C. Li, “Graphene quantum dots: emergent nanolights for bioimaging, sensors, catalysis and photovoltaic devices," Chemical Communications, vol. 48, no. 31, pp. 3686-3699, 2012.

[16] J. Qiu, R. Zhang, J. Li et al., "Fluorescent graphene quantum dots as traceable, $\mathrm{pH}$-sensitive drug delivery systems," International Journal of Nanomedicine, vol. 10, pp. 6709-6724, 2015.

[17] A. D. Güçlü, P. Potasz, M. Korkusinski, and P. Hawrylak, Graphene Quantum Dots, Springer-Verlag, Berlin, Germany, 2014.

[18] R. Shimano, G. Yumoto, J. Y. Yoo et al., "Quantum Faraday and Kerr rotations in graphene," Nature Communications, vol. 4, p. 1841, 2013.

[19] A. Narita, X. Y. Wang, V. Feng, and K. Müllen, "New advances in nanographene chemistry," Chemical Society Reviews, vol. 44, no. 18, pp. 6616-6643, 2015.

[20] A. B. Bourlinos, A. Stassinopoulos, D. Anglos, R. Zboril, M. Karakassides, and E. P. Giannelis, "Surface functionalized carbogenic quantum dots,” Small, vol. 4, no. 4, pp. 455-458, 2008.

[21] S. Kim, S. W. Hwang, M.-K. Kim et al., "Anomalous behaviors of visible luminescence from graphene quantum dots: interplay between size and shape," ACS Nano, vol. 6, no. 9, pp. 8203-8208, 2012.

[22] F. Xu, L. F. Gerlein, X. Ma, C. R. Haughn, M. F. Doty, and S. G. Cloutier, "Impact of different surface ligands on the optical properties of $\mathrm{PbS}$ quantum dot solids," Materials, vol. 8, no. 4, pp. 1858-1870, 2015.

[23] K. Yang, L. Feng, X. Shi, and Z. Liu, "Nano-graphene in biomedicine: theranostic applications," Chemical Society Reviews, vol. 42, no. 2, pp. 530-547, 2013.

[24] C. Kittel, Quantum Theory of Solid, John-Wiley \& Son Inc., New York, NY, USA, 1963.

[25] M. Hasanzadeh, A. Karimzadeh, N. Shadjou et al., "Graphene quantum dots decorated with magnetic nanoparticles: synthesis, electrodeposition, characterization and application as an electrochemical sensor towards determination of some amino acids at physiological $\mathrm{pH}$," Materials Science and Engineering: C, vol. 68, pp. 814-830, 2016.

[26] A. Candini, S. Lumetti, C. Godfrin et al., "Addressing a single molecular spin with graphene-based nanoarchitectures," in Advances in Atom and Single Molecule Machines, pp. 165-184, Springer, Berlin, Germany, 2017.

[27] Y. Sun, Y. Zheng, H. Pan et al., "Magnetism of graphene quantum dots," npj Quantum Materials, vol. 2, no. 1, pp. 1-7, 2017.

[28] R. Justin, K. Tao, S. Román et al., "Photoluminescent and superparamagnetic reduced graphene oxide-iron oxide quantum dots for dual-modality imaging, drug delivery and photothermal therapy," Carbon, vol. 97, pp. 54-70, 2016.

[29] N. Alegreta, A. Criadoa, and M. Pratoto, "Recent advances of graphene-based hybrids with magnetic nanoparticles for biomedical applications," Current Medicinal Chemistry, vol. 24, no. 5, pp. 529-536, 2017.

[30] Z. L. Wu, M. X. Gao, T. T. Wang, X. Y. Wan, L. L. Zheng, and C. Z. Huang, "A general quantitative $\mathrm{pH}$ sensor developed with dicyandiamide $\mathrm{N}$-doped high quantum yield graphene quantum dots," Nanoscale, vol. 6, no. 7, pp. 3868-3874, 2014. 


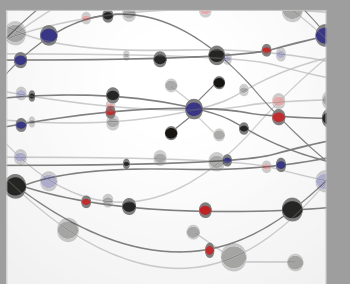

The Scientific World Journal
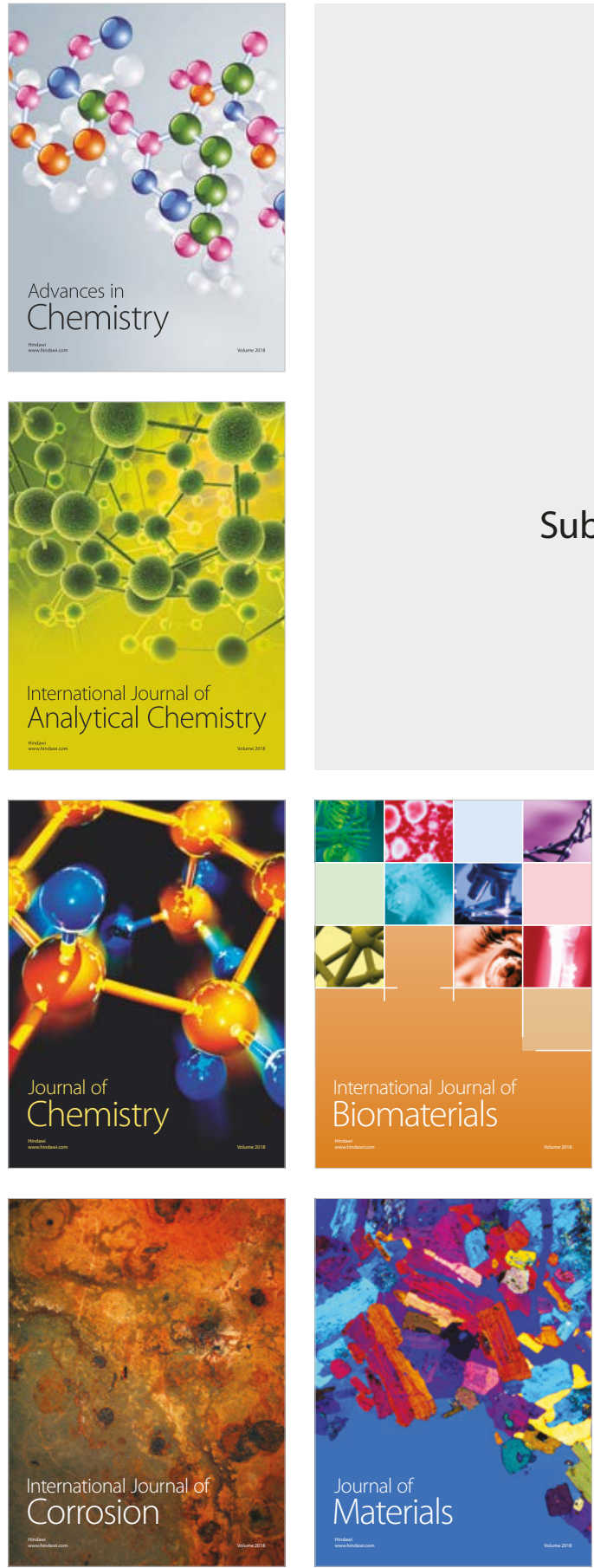

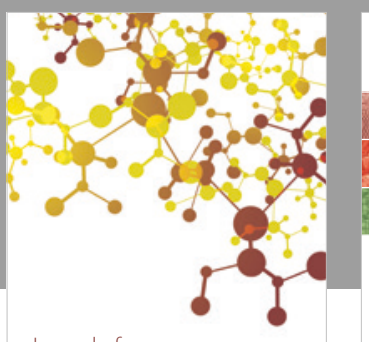

Journal of

Applied Chemistry
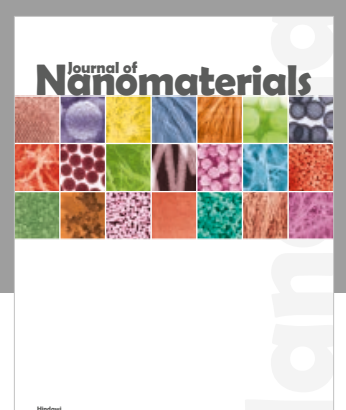

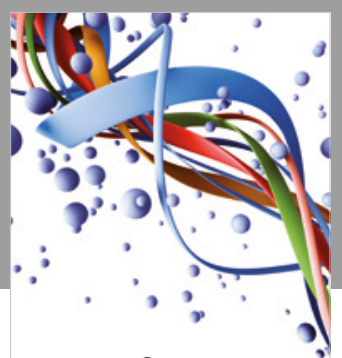

Scientifica

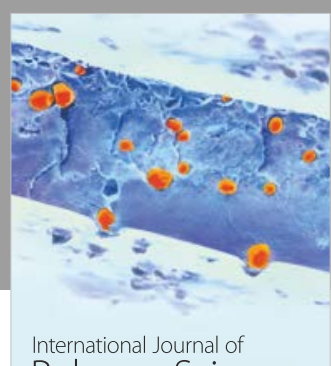

Polymer Science

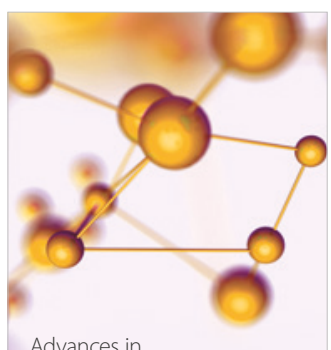

Physical Chemistry
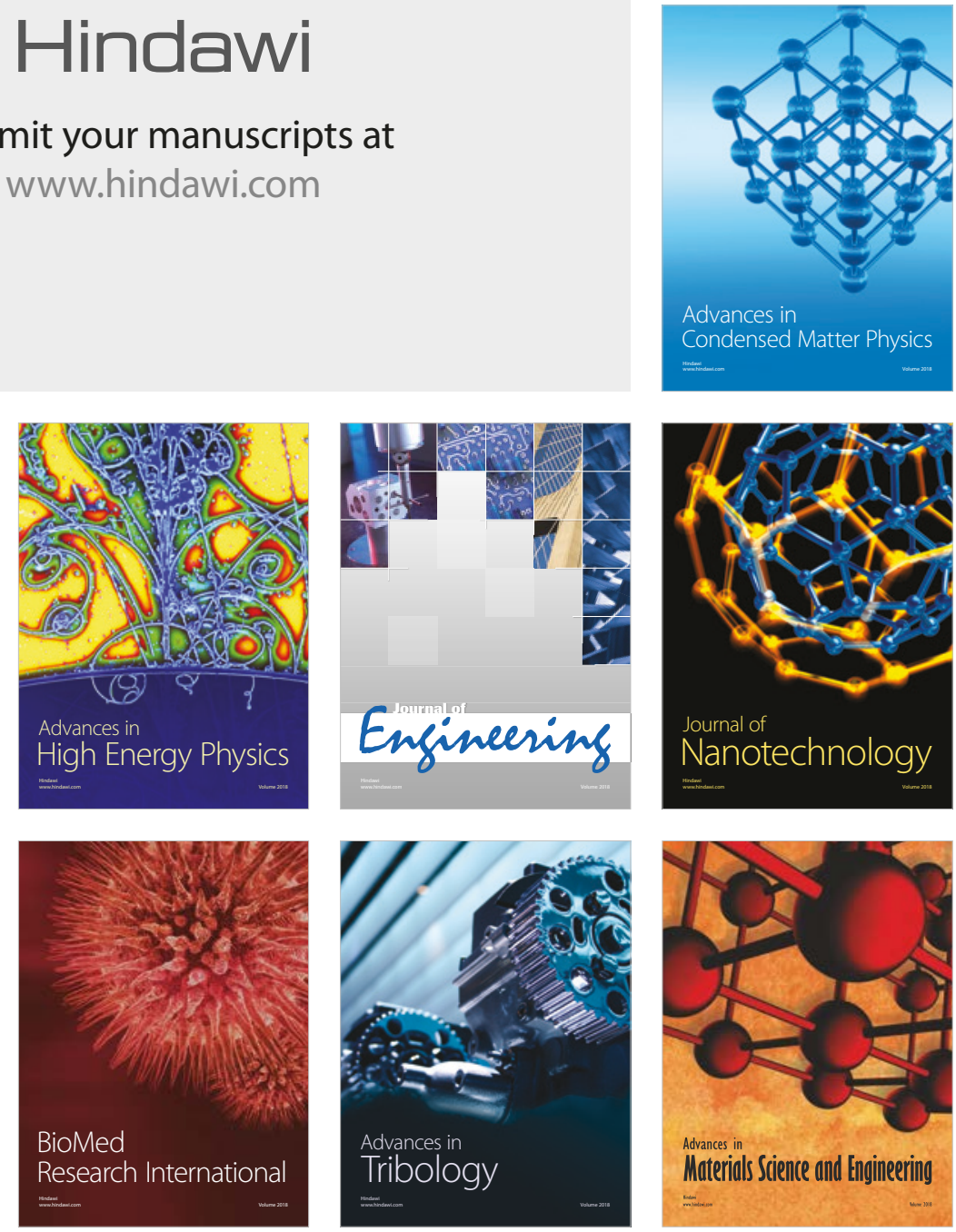